\title{
Kant en tant que Philosophe de la Science de la Nature
}

\author{
[Kant como um Filósofo da Ciência da Natureza]
}

\author{
Pedro M. S. Alvest
}

Resumé: Nous commençons par souligner les trois innovations kantiennes qui mettent la philosophie théorique sur une nouvelle voie. Premièrement, une nouvelle forme de la Philosophie théorique, présentée non plus comme une théorie directe de la nature, mais comme une enquête réflexive de la science mathématique de la Nature comme un fait primitif. Deuxièmement, la méthode transcendantale, comme détermination réflexive-régressive des conditions subjectives de possibilité de la connaissance des objets en général. Troisièmement, le rôle central joué par la science newtonienne de la nature. Après cela, je discute de la réévaluation logico-positiviste du récit de Kant à la lumière de la relativité générale d'Einstein et de la réplique de Cassirer, soulignant avec ce dernier la nécessité d'une révision des thèses de Kant sur l'espace, sans toutefois abandonner la posture transcendantale kantienne et sa théorie de la constitution de quelque chose comme objet de connaissance. Enfin, nous concluons par quelques remarques qui visent à une esthétique transcendantale renouvelée qui serait capable d'absorber la théorie de l'espace-temps que la physique d'Einstein a mise en avant.

Mots-clés: Kant. Cassirer. Philosophie de la Nature. Théorie de la Connaissance. Espace.

Resumo: Começo enfatizando as três inovações kantianas que colocam a filosofia teórica em um novo caminho. Em primeiro lugar, uma nova forma para a Filosofia teórica, apresentada nao mais como uma teoria direta da natureza, mas como uma investigação reflexiva da ciência matemática da Natureza como um fato primitivo. Em segundo lugar, o método transcendental, como determinação reflexivo-regressiva das condições subjetivas de possibilidade do conhecimento dos objetos em geral. Em terceiro lugar, o papel central desempenhado pela ciência newtoniana da natureza. Em seguida, discuto a reavaliação lógico-positivista do relato de Kant a luz da relatividade geral de Einstein e da réplica de Cassirer, enfatizando com este último a necessidade de uma revisão das teses de Kant sobre o espaço, sem abandonar, no entanto, a postura transcendental kantiana e sua teoria da constituição de algo como objeto de conhecimento. Por fim, concluo com algumas observações que visam uma estética transcendental renovada que seria capaz de absorver a teoria do espaço-tempo que a física de Einstein propôs.

Palavras-chave: Kant. Cassirer. Filosofia da Natureza. Teoria do Conhecimento. Espaço.

${ }^{*} \mathrm{PhD}$, Associate Professor with tenure at the Department of Philosophy of the Faculty of Arts of the University of Lisbon. Researcher of the Center of Philosophy of the University of Lisbon. Editor of Phainomenon - A Journal of Phenomenological Philosophy. E-mail: psalves2@gmail.com ORCID: https://orcid.org/0000-0001-5259-9367. 


\section{Liminaires : les trois «innovations» kantiennes}

La Critique de la raison pure, l'ouvre majeure de Kant, a été interprétée de plu-sieurs points de vue tout a fait incompatibles. Cette pluralité d'interprétations d'ensemble a commencé au temps de Kant lui-meme et, depuis lors, elle s'est développée sans cesse en des visions toujours nouvelles sur la teneur et le but final de la Critique. Il en résulte qu'on n'est meme pas tout a fait d'accord sur ce que signifie, aux yeux de Kant lui-meme, le projet d'une critique de la raison. De Reinhold, de Beck ou de Fichte jusqu'a Cassirer et Heidegger, on peut trouver maintes interprétations non seulement différentes, mais aussi qui a peine partagent un langage commun.

Je ne veux pas discuter ici ni de la pertinence ni meme de la justesse de quelques-unes d'entre elles, lorsque comparées avec les déclarations explicites de Kant sur les objectifs et la signification de son ouvre théorique.

Mon idée de base est que Kant, apres s'etre dédié a certains problemes fondamentaux dans le champ de la Philosophie Naturelle, dans la période nommée "pré-cri-tique »- tels que la contradiction apparente, dans le concept de matiere, entre sa structure corpusculaire et la divisibilité infinie de l'espace; la querelle leibnizienne des forces vives et de la nature de la substance; le caractere réal ou idéal, relatif ou ab- solu de l'espace et la validité du principe des indiscernables; aussi la question du mouvement absolu, le principe de la continuité et d'autres questions encore - mon idée, je répete, c'est que Kant, apres avoir discuté des questions conceptuelles concernant les fondements de la Physique, dans un contexte de confrontation entre la métaphysique leibnizo-wolffienne et la physique expérimentale de Newton, introduit trois changements décisifs qui, a eux seuls, ont produit quelque chose comme un «âge kantien » dans la réflexion philosophique sur les sciences de la nature. C'est donc essentiellement en tant que philosophe de la science de la nature que je vois le Kant de la Critique de la raison pure.

\section{Premiere innovation : une forme nouvelle pour la philosophie théori- que}

Premier changement. Kant découvre une forme nouvelle de philosophie, qu'il appelle justement philosophie transcendantale, laquelle n'est pas une connaissance d'objets en particulier, mais une exhibition des conditions subjectives qui rendent possible la connaissance d'objets en général. Ainsi, la philosophie transcendantale sera un savoir de second niveau. Elle n'est pas le systeme des sciences de la Nature, elle n'est pas aussi une discussion sur les concepts fondamentaux des scien- 
ces empiriques ou des sciences pures. La philosophie transcendantale sera l'explicitation du mode comme il sera possible qu'il y ait, en général, une conscience et une science de la Nature. On sait que Kant distingue soigneusement les conditions relatives a la donation intuitive, c'est-a-dire, les conditions esthétiques, et ensuite les conditions logiques, relatives a la possibilité de penser des objets en général, et, finalement, qu'il renvoie les conditions logiques et esthétiques a l'unité de l'aperception, a l'unité du sujet épistémique, en tant que condition ultime de possibilité de la connaissance objective en général. Ainsi, la philosophie transcendantale mettra a découvert le caractere aprioristique de certaines représentations et montrera comment elles rendent possible ou comment elles sont la source d'ou jaillit la connaissance d'objets de premier niveau, qu'ils soient les objets des sciences empiriques ou qu'ils soient les objets des sciences pures, comme la géométrie et l'arithmétique.

Simultanément - ce qui, a mon avis, n'a jamais été convenablement remarqué - la philosophie transcendantale que Kant développe dans la Critique ne contient seulement une théorie sur ce qui est un objet de connaissance possible, mais aussi une théorie sur les pseudo-objets et la connaissance apparente qui se réfere a eux. Nous pouvons trouver la théorie kantienne de l'objet de connaissance possible dans
l'Esthétique et dans l'Analytique. La Dialectique, au contraire, est une théorie des conditions qui rendent possible (et inévitable) la construction de pseudo-objets (Kant les nommera " objets hyperboliques », excessifs) et la parution d'une illusion générale sur l'extension illimitée de notre connaissance. En effet, la Dialectique montre comment, en partant des processus de la connaissance objective et a cause de ces processus memes (notamment, selon Kant, les syntheses des catégories de la relation) se profilent a l'horizon des objets hyperboliques qui donnent l'illusion d'un rapport cognitif qui dépasse le terrain de l'expérience possible.

Il y a deux aspects qui, a mon sens, sont importantes dans cette théorie kantienne des pseudo-objets. D'abord, elle est intimement liée a la théorie des objets de connaissance possible. Les pseudo-objets ne son autre chose que, (i), des représentations de la totalité des syntheses qui constituent les objets de connaissance et (ii) un changement de point de vue, qui mene a considérer ces représentations de la totalité comme de nouveaux concepts de choses qui ne sont plus référées a une intuition possible, mais qui sont " en-soi », c'est-a-dire, qui sont posées comme si elles étaient des réalités indépendantes des processus cognitifs de constitution objective. On pourrait faire une théorie plus vaste des processus de construction hyperbolique d'unités objectives. Son principe général sera quelque chose comme le 
suivant : chaque fois que l'unité objective est posé non pas par référence a un acte déterminé de synthese et, par conséquence, par référence au pont de vue du sujet épistémique, mais qu'elle est posée comme étant "en soi », dans la chose meme, indépendamment des processus de la connaissance, on aura devant nous un pseudo-objet, c'est-adire, une unité dont la structure n'a pas été constitué par des actes de la pensée objectivante. En ce sens-la, on pourrait dire qu'il y a nombreux pseudoobjets et pas seulement les trois objets inconditionnés de la Dialectique. C'est justement a cause de cela que, deuxiemement, la critique du concept wolffien de Métaphysique Spéciale, avec les trois disciplines de Psychologie Rationnelle, de Cosmologie Rationnelle et de Théologie Naturelle, n'est, a mon avis, que l'application a un cas particulier d'une théorie générale des pseudoobjets qu'on pourrait bâtir ayant pour base les principes kantiens. Cette théorie des pseudo-objets est, dans la Critique, limitée a son application au cas de la Métaphysique Spéciale. Une théorie générale des pseudo-objets, qui est une des grandes idées kantiennes qui n'ont jamais été assez explicitées, est encore largement a construire.

En somme, comme Michael Friedman l'a remarqué (2001, p.8), le concept de philosophie transcendantale vient se substituer a ce qui était le concept traditionnel, de souche cartésienne, de philosophie, partagé en philosophie naturelle ou physique et philosophie premiere, ou métaphysique, englobant chacune d'elles les sciences de la partie corporelle et de la partie incorporelle de la réalité, c'est-a-dire, Dieu et l'âme. D'autre part, et c'est quelque chose a laquelle Michael Friedman ne semble pas porter d'attention, la philosophie transcendantale, définie comme un savoir concernant les conditions qui rendent possible la connaissance des objets de premier ordre, est aussi une théorie de la façon dont la connaissance d'objets sécrete des pseudo-objets et l'illusion d'un autre type de connaissance qui se rapporte a eux.

\section{Deuxieme innovation : la façon de construire la science de la Nature}

Second changement. Kant développe des arguments purement gnoséologiques, et non plus de teneur métaphysique, pour montrer pourquoi l'espace et le temps sont des conditions universelles des objets de connaissance possible; en complémentarité, Kant produit, dans le célebre chapitre de la Déduction Transcendantale des Concepts Purs, une preuve, elle aussi purement gnoséologique, de la raison par laquelle tout ce qui peut etre donné dans l'espace et dans le temps, comme objet d'une intuition possible, sera nécessairement soumis aux concepts purs de l'entendement et sera, donc, toujours pensable par eux. En un mot, Kant découvre une façon 
entierement nouvelle de prouver des énoncés sur la structure des objets que nous pouvons connaître. Il parle non directement des choses et de la Nature, mais de notre conscience des choses et de la Nature, en tant que cette conscience est une conscience cognitive et non pas, par exemple, esthétique ou autre. On pourrait dire que la position d'une réalité ou d'une Nature suppose la position préalable d'une conscience possible de réalité ou d'une Nature formaliter spectata. C'est a cette conscience que Kant s'intéresse. On appellera par apres cette nouvelle façon de réfléchir une Erkenntnistheorie, une théorie de la connaissance ou gnoséologie, laquelle est, en vérité, une théorie de l'etre en tant que connu.

Avec cette double démonstration strictement gnoséologique, centrée sur les formes subjectives de l'intuition et sur la relation nécessaire du divers de l'intuition a l'unité objective de la conscience, donc a la synthese catégoriale, Kant prétend avoir résolu le probleme de la connaissance d'expérience et, par l'entremise des catégories schématisées, c'est-a-dire, de l'Analytique des Principes, avoir rencontré les fondements de la science mathématique de la Nature que Newton avait développée et qui est, pour lui, le modele de la Philosophie Naturelle. A la fin de l'Esthétique, nous possédons un ensemble de raisons qui nous permettent de dire que tout objet s'individualise sous la forme d'un ensemble de localisations dans l'espace et dans le temps. Ainsi, il faut qu'un objet de connaissance possible commence par etre représentable comme une trajectoire ou un parcours dans l'espace et le temps. Quoique Kant ne l'explicite pas dans la Critique, les objets de connaissance possible, étant individualisés par des trajectoires qui leur sont propres dans l'espace et le temps, permettent de vérifier certaines propriétés tres bien définies. Par exemple, aucun objet n'est superposable a un autre, c'est-adire, aucun objet ne peut avoir la meme localisation spatio-temporelle qu'un autre objet différent, ce qui correspond, physiquement, a l'impénétrabilité, qui est une des propriétés de la matiere, comme Kant l'affirme des sa Monadologie Physique (en la dérivant du concept de force répulsive). Une autre propriété remarquable (que la physique du $\mathrm{XX}^{\text {eme }}$ siecle a mis en doute) est celle de la continuité, par laquelle on affirme, en substance, que, entre deux positions différentes d'un objet dans l'espace en deux temps, on doit pouvoir trouver toutes ses positions intermédiaires contiguës avec le degré de précision que on voudra et en admettant une vitesse qui peut s'accroître a l'infini, sous peine de ne pas pouvoir parler d'un seul ou du meme objet. A la fin de l'Esthétique, nous voyons aussi la raison qui fait que et la Géométrie et le Calcul soient applicables aux objets qui nous sont donnés : c'est que, par le fait meme qu'ils nous sont donnés, ils ont des localisations spatio-temporelles et des trajec- 
toires continues dans l'espace et dans le temps, avec de vitesses changeant en fonction des forces appliqués. Cela s'avere déterminant pour comprendre la possibilité de la Mécanique de Newton et l'utilisation qu'elle fait soit de la Géométrie soit de concepts comme celui d'accélération, qui ne peut etre défini mathématiquement qu'a partir du Calcul Différentiel (il s'agit de la dérivée de deuxieme ordre de l'incrément de la vitesse par l'incrément du temps). D'autre part, a la fin du Systeme des Principes, notamment des trois analogies de l'expérience, on comprend la raison ultime des trois Lois du Mouvement de la Mécanique de Newton, qui sont fondées par le moyen des concepts de substance, de force active et d'action réciproque ou comercium (Gemeinschaft, communauté) entre les substances, et pas seulement de " coexistence », a la maniere leibnizienne. En fait, le rôle que les catégories de la relation jouent dans la fondation des trois lois de Newton est peut reconnut par les commentateurs, mais tout a fait visible depuis le Duisburgsche Nachlass, de 1775. En effet, la on peut constater que, avant de découvrir le Leitfaden et, par conséquence, la dérivation logique des catégories en partant des jugements, c'est la triplicité des lois de Newton (et pas les formes catégorique, hypothétique et disjonctive du jugement) qui détermine les réflexions de Kant sur la «causalité», la «substance» et la «réciprocité».

Dans son travail de fondation de la science de la Nature, Kant a compris donc qu'il y a, dans les sciences empiriques, deux types d'énoncés. Ceux qui portent sur les objets donnés; et d'autres qui constituent les objets memes sur lesquels les premiers di-sent quelque chose. Contre la dualité de Hume de l'analytique et du synthétique a poste-riori, Kant découvre les jugements synthétiques a priori et attribue a eux un rôle constructif de l'objet de l'expérience possible. Il y aura donc, pour Kant, une stricte hiérarchie des sciences. La loi de la gravitation, par exemple, est une loi empirique et exprime une connaissance d'expérience. Toutefois, la loi newtonienne de la gravitation ne serait pas formulable sans les notions d'inertie et de taux de variation instantanée de la vitesse de deux masses l'une par rapport a l'autre par l'action d'une force (c'est-a-dire, la notion mathématicophysique d'accélération), notions qui, plus que matieres empiriques discernables dans l'expérience (l'inertie est déja une situation idéale - Newton l'a définie par rapport a l'espace et au temps absolus), sont des lois idéales qui structurent les apparences et donnent la base aux lois empiriques de la mécanique. Ce sont justement les "lois du mouvement" des Principia Mathematica Philosophia Naturalis, notamment les deux premieres, que Kant englobe dans la rubrique nommée "théorie générale du mouvement". D'autre part, la notion meme d'accélération, qui signi- 
fie le taux de variation instantanée de la vitesse, est informulable sans l'outil mathématique du cal-cul différentiel ou du calcul des "fluxions", dans le langage de Newton. Les lois pures du mouvement (inertie, force nette imprimée, égalité d'action et de réaction), le calcul différentiel et les théoremes géométriques sont donc, plus que connaissances d'objets discernables dans l'expérience, des structures conceptuelles qui permettent d'organiser les perceptions dans une expérience d'objet et de déterminer les lois empiriques pertinentes, dans ce cas, la loi d'attraction des corps en raison directe des masses et en raison inverse du carré des distances. Sans ces notions, la loi meme de la gravitation ne serait pas formulable et le " phénomene » gravitationnel ne serait meme pas visible en tant que tel. ${ }^{1}$

Transformer des jugements de perception en des jugements d'expérience, en établissant la façon dont la perception doit etre différenciée et organisée dans une expérience d'objet - voila la force et l'efficacité des principes constitutifs. Et voici le sens fort de la notion d'un principe constitutif synthétique a priori. Kant le pense comme l'anticipation méthodique d'une expérience possible, de telle façon que la construction de cette expérience (en- core sans contenu empirique), selon les concepts purs et les outils mathématiques correspondants, par référence a ce qu'il désigne comme l'intuition pure (de l'espace et du temps) c'est le lieu de la validation de ces connaissances a priori. La construction anticipatrice de l'expérience possible est la source de connaissances synthétiques a priori, précisément, de connaissances sur ce que signifie etre un objet de connaissance. La science de l'espace et la Mathématique pure, les catégories et le systeme des premiers principes de la Science de la Nature sont, aux yeux de Kant, des connaissances de ce genre.

\section{Troisieme innovation : le rôle fon- damental de la Physique newtonienne}

En dernier lieu, il y a un troiseme changement a tout titre décisif. Quelque chose qui est, a mon sens, pleine d'importance c'est le fait que Kant montre, dans la Critique de la raison pure, que le langage de la Physique est le dernier mot sur le monde des objets empiriques. Dans la Monadologie de Leibniz, on trouve l'idée d'un monde de substances simples non-spatiales et non-temporelles, par rapport auquel la représentation d'un monde de corps

\footnotetext{
${ }^{1}$ On ne parle pas du phénomène sensible de la chute des graves en direction du centre de la Terre, phénomène observable par la perception commune et que peut être "expliqué" d'une multiplicité de façons différentes. On parle plutôt de la conception selon laquelle la chute des graves est un cas très spécial d'une loi générale d'attraction universelle entre les masses, loi que rende raison tant de la chute des pommes comme du mouvement des planètes autour du Soleil, mouvement qu'on ne peut pas voir immédiatement par l'entremise des organes de la perception commune, mais qu'on peut seul établir théoriquement.
} 
dans l'espace et dans le temps était une représentation sans doute bien fondée, mais non pas une représentation adéquate. Un entendement infini pourrait calculer l'ensemble des relations d'ordre entre les substances, la compossibilité de ses propriétés et les relations de dépendance entre elles a partir du principe de raison suffisante et sans faire intervenir le concept de corps, de position dans l'espace et dans le temps et d'influence réelle entre les substances. Le langage de la Physique n'était donc pas, pour un leibnizien, l'ultima ratio sur la réalité. La Physique avait sa raison dans la Métaphysique et empruntait d'elle ces concepts fondamentaux. Néanmoins, avec Kant, on trouve justement l'idée que le langage de la Physique, c'est-a-dire, ses concepts et ses méthodes mathématiques ne sont pas un langage provisoire sur un monde d'apparences sensibles opposé aux essences, mais la seule forme d'intelligibilité possible du monde des phénomenes, que Kant appelle Erscheinungen, apparitions, qui ne s'opposent pas a une autre chose sinon au vide de la connaissance des choses en-soi. Le monde sensible ne renvoie plus a un monde intelligible, mais, en tant que monde de l'apparaître, il renvoie aux conditions subjectives de la possibilité de l'apparition, c'est-a-dire, aux formes a priori de l'intuition.

En somme, j'ai voulu dire que le Kant de la Critique est le Kant qui cherche les fondements de la physique, telle que Newton l'avait établie, en montrant comment un sujet épistémique aura devant soi comme objets empiriques justement une Nature qui sera connaissable seulement par une science telle que la physique newtonienne : une Nature composée d'une multiplicité de substances, qui remplissent l'espace ( substantia phaenomenon, c'est-a-dire matiere) et se conservent (dans son quantum) au long du temps, et qui sont en rapports d'action réciproque, selon l'ordre de la simultanéité, et d'influx causal, selon l'ordre de la succession. Au meme temps, il y a certainement une diversité d'autres problemes qui gravitent autour de celui-ci. Je me réfere au probleme humean de la causalité et de la connaissance d'expérience, que Kant a résolu par sa déduction transcendantale; je me réfere aussi au probleme de la table complete des concepts purs, que, selon une suggestion de Vleeschauwer (1976, p. 223), provient surtout d'une lecture des Nouveaux Essais, de Leibniz, et que Kant a résolu, de la forme maladroite qu'on sait, par sa déduction métaphysique, en partant de la table des jugements; il y a encore le probleme conceptuel de l'existence, provenant de Crusius. Mais tout ça se trouve conditionné par le probleme fondamental de Kant - celui d'une théorie de l'objet de la connaissance possible (celui de la Physique) et du pseudo-objet de connaissance illusoire (celui de la Métaphysique).

Malgré cela, a mon avis, la façon kan- 
tienne de poser les questions gnoséologiques est périmée. Kant ne se libere pas completement du programme empiriste d'une science de la nature humaine. Il parle partout des facultés de connaissance. Et, en rigueur, ses preuves gnoséologiques veulent mettre a découvert la soi-disant structure des facultés de l'intuition et de la pensée qui appartient a nous, hommes, et qui sera, par-la, immuable. Ainsi, l'espace de notre connaissance physique sera toujours l'espace euclidien de notre faculté d'intuition. Et les principes constitutifs de l'objet d'expérience seront toujours les memes, par la simple raison que les catégories seront les formes immuables de la spontanéité de nôtre entendement. Cette conception, fixée sur une prétendue " nature » des facultés de la connaissance, a conduit Kant a une conception du synthétique $a$ priori comme un principe universel et apodictique, donc absolument immuable. Mais c'est justement cette immutabilité des principes constitutifs que le développement de la science physique au $\mathrm{XX}{ }^{\text {eme }}$ siecle a contredite.

Néanmoins, on peut trouver une autre perspective plus intéressante chez Kant. La Critique balance toujours entre deux extremes - d'un côté, il $\mathrm{y}$ a justement cette vision statique et stratifiée des facultés de connaître; mais, de l'autre, il y a aussi une vision dynamique et constructive de l'acte synthétique en tant que processus d'objectivation. On pourrait oublier désormais les idées kantiennes sur la nature et la structure des facultés et définir le synthétique a priori comme étant tout énoncé que, au lieu de porter sur les objets de l'expérience, construit les objets memes de que la science empirique va parler. A la lumiere de cette conception, il y aura toujours des principes synthétiques a priori, mais ces principes ne seront jamais immuables. Pour la mécanique de Newton, la géométrie euclidienne, le calcul, les lois d'inertie et de conservation de la masse seront de tels principes. Mais, par exemple, pour la relativité restreinte d'Einstein, on aura comme principes constitutifs la géométrie quadridimensionnelle pseudo-euclidienne de Minkowski et le constant $c$ comme vélocité maximale de la propagation des signaux dans le vide. La relativité généralisée aura d'autres principes (" synthétiques a priori ») encore, notamment le calcul tensoriel.

En un mot, on aura toujours une science avec des principes constitutifs, contre tous les empirismes, de David Hume jusqu'a Quine. Cela serait le grand enseignement de la gnoséologie de Kant. Et cet enseignement est encore actuel. Faut-il parler d'un a priori révisable et pas immuable? Ça ne sera pas kantien selon la lettre, certainement. Mais, a mon sens, cela serait la seule façon de rester encore kantien aujourd'hui. 


\section{Une lecture néo-kantienne de la re- lativité d'Einstein}

Plus encore que, par exemple, la phénoménologie, qui était une philosophie naissante au temps des essais de Einstein de 1905, sur la relativité restreinte, et de 1915, sur la relativité généralisée, la philosophie transcendantale de Kant - un patrimoine intellectuel affermi et renforcé par le néo-kantisme - a été immédiatement mise en question en ce qui concerne ses présupposés fondamentaux par le surgissement de la nouvelle physique de la relativité. Moritz Schlick et Hans Reichenbach ont été deux figures majeures en cette confrontation, qui se développe surtout a partir des années vingt du vingtieme siecle. La contestation du "synthétique a priori ", en tant que principe constitutif de l'objectivité d'expérience, substitue par le principe de la "définition coordinatrice » (these originaire de Schlick), lequel permet un empirisme avec des principes constitutifs, mais sans compromis avec l'universalité et l'apodicticité au sens kantien, ${ }^{2}$ aussi que bien que l'exhibition des insuffisances de la doctrine kantienne de l'espace et du temps - lesquels, selon Einstein,
« auraient perdu l'ultime reste de réalité objective »- surgissent comme les deux axes principaux de cette évaluation négative des capacités de la philosophie critique a incorporer les résultats de la nouvelle physique. "C'est a ce processus de dissolution du synthétique a priori que nous devrons incorporer la théorie de la relativité, quand nous désirons la juger au point de vue de l'histoire de la philosophie. " (REICHENBACH, 2000, p. 307-308) $)^{3}$ Cette affirmation de Reichenbach, en 1949, peut nous donner une idée précise de ce qui a été le centre du débat au début des années vingt.

Dans ce contexte, l'essai de 1921 de Ernst Cassirer [paru presque en meme temps que l'essai de Reichenbach (1920)], intitulé Sur la théorie de la relativité einsteinienne - considérations gnoséologiques, ${ }^{4}$ réalise un mouvement en direction opposée. Cassirer n'y essaie pas une assimilation des theses de la nouvelle physique par le kantisme classique, travail qui serait vain, mais plutôt une réélaboration de la philosophie critique, tâchant de montrer comment et en quelle mesure la nouvelle physique représente un approfondissement de quelques theses fonda-

\footnotetext{
2"Between the two [Mach and Kant] remains standing the empiricist view, according to which these constitutive principles are either hypotheses or conventions; in the first case they are not a priori (since they lack apodeicticity), and in the second they are not synthetic. [...] A thinker who in general perceives the unavoidability of constitutive principles for scientific experience should not yet on that account be designated a critical philosopher. An empiricist can, for example, very well recognize the presence of such principles; he will only deny that they are synthetic and a priori in the sense described above." (SCHLICK, 1979, p.324)

3 "It is this process of a dissolution of the synthetic a priori into which we must incorporate the theory of relativity, when we desire to judge it from the viewpoint of the history of philosophy."

${ }^{4}$ Ernst Cassirer - Zur Einsteinschen Relativitätstheorie. Erkenntnistheoretische Betrachtungen., 1921. Republié en Zur modernen Physik., 1994, pp. 1-125 (on citera d'après cette dernière édition).
} 
mentales du criticisme en ce qui concerne la constitution de l'objectivité d'expérience.

A ce propos, la premiere chose a faire sera de délier la philosophie critique de ses attaches a la physique newtonienne. Si celle-ci représente une phase définitivement dépassée de la connaissance de la réalité physique, la philosophie critique, de son côté, en tant que recherche sur le processus d'objectivation qui pose devant le physicien quelque chose comme une réalité physique donnée dans l'expérience, doit pouvoir survivre au moment de la connaissance de la Nature auquel elle a été temporairement liée et perdurer par-dela de lui. En cela se décide l'actualité de la philosophie critique, et c'est justement celle-la la question fondamentale de l'essai de Cassirer. "Si Kant croyait posséder dans l'ouvre fondamentale de Newton [...] quelque chose comme un codex assuré de la vérité » physique, [...] la relation entre philosophie et science exacte qu'il acceptait a changé depuis lors » (CASSIRER, 1994, p. 5). Les enjeux que, selon Cassirer, signalent l'obsolescence de la physique newtonienne sont " le fait de la géométrie », c'est a dire, le développement, depuis Gauss, des géométries non-euclidiennes et son usage dans la théorie physique par la relativité généralisé ; le "systeme de la mécanique classique ", non seulement réduit a une théorie plus générale, mais aussi outrepassé par le concept de « champ », pro- venu de la théorie électromagnétique de Faraday et de Maxwell ; et, finalement, la question de fond » concernant l' " essence » de l'espace et du temps, la question disputée depuis Leibniz et Clarke. Comme Cassirer se demande, Si Kant ne voulait etre autre chose que le systématisateur philosophique de la science newtonienne de la Nature, ne restera, donc, sa doctrine liée aussi au destin de la physique newtonienne, et ne devront tous les changements qu'elle a souffert agir aussi en retour immédiatement sur la configuration des doctrines fondamentales de la philosophie critique?

On sait bien que la philosophie kantienne a voulu donner la justification ultime pour chacun de ses aspects de la science mathématique de la Nature, de Newton. L'intuition pure de l'espace, mise a la base de la science géométrique, assure son caractere apriorique (contre certaines affirmations de Newton lui-meme), mais la compromet, d'une façon apparemment irrémédiable, avec la géométrie Euclidienne; les analogies de l'expérience, de l'analytique des principes, suivent et décalquent les trois lois des Principia Mathematica Philosophia Naturalis (soit les principes de la conservation de la masse, de la causalité et de l'action réciproque, lesquels répondent aux lois newtoniennes de l'inertie, de la force et de l'égalité de l'action et de la réaction); en ce qui regarde la question de l'espace et du temps en tant que tels, si 
Kant prend des distances soit par rapport a Leibniz soit a Newton avec sa théorie de l'idéalité transcendantale et de la réalité empirique, la these selon laquelle l'espace et le temps sont des intuitions pures (ou formes d'intuition) le mene a défendre qu'il s'agit, en premier lieu, de deux représentations séparées, et, de suite, qu'il y a une unité primitive de chacune de ces représentations (l'espace et le temps, chacune d'elles étant une singularis repraesentatio), que les espaces et les temps s'obtiennent donc par limitation de cette représentation unitaire en tant que ces parties, nécessairement coordonnés dans une représentation globale une et unique.

Tous ces aspects sont des points de doctrine relativement auxquels le développement historique des sciences exactes, et de la physique en particulier, a décidé contre les theses de la Critique. Cependant, comme Cassirer le souligne, l'ouvre de Kant n'est pas un compendium de l'état de la science courante a son temps, mais une enquete gnoséologique sur les sources de la connaissance et la constitution de l'objectivité d'expérience, une recherche qui emploie les sciences qui lui sont contemporaines seulement comme un matériel pour ces recherches. «Nous ne connaissons pas «les objets »; [...] nous construisons une connaissance de l'objet.» (CASSIRER, 1977, p. 343)
- c'est ce processus ou cette fonction d'objectivation que la critique de la connaissance s'efforce pour mettre en lumiere. En ce sens, pour Cassirer, la tâche de la philosophie transcendantale consiste a exhiber les invariantes logiques ultimes, communs a toutes les formes possibles de théories, en tant qu'éléments constitutifs nécessaires. Celle-ci n'est pas une tâche qui soit réalisable en quelque phase historique de la pensée scientifique. Mais la «fonction objectivante » ou la « loi supreme d'objectivation », que la recherche gnoséologique découvre, doit fonctionner comme une exigence de l'entendement qui fixe une direction pour " le développement continu du systeme de l'expérience.» ${ }^{6}$ Non seulement l'a priori est, donc, une dynamique d'objectivation constamment en devenir, que se réalise imparfaitement et qui s'approfondi en chaque étape de la science de la Nature, mais aussi la division étanche entre science de la Nature et critique de la connaissance s'avere, aux yeux de Cassirer, comme une séparation artificielle. Cassirer l'affirme presque au début de son essai : " un regard attentif sur l'histoire de la physique nous enseigne justement que ces progres les plus importants ont en général une connexion intime avec des considérations d'ordre gnoséologique. » (CASSIRER, 1994, p. 6) On peut

\footnotetext{
${ }^{5}$ (Nous soulignons).

${ }^{6}$ A propos de cet a priori relativisé, voire Thomas Ryckman (2005, p. 13 e sgs).
} 
voir cela chez Galilée, Kepler, Newton, et on peut le voir d'une forme encore plus éclatante a propos de la reformulation einsteinienne du principe de la relativité, parce que Einstein, « en particulier afin de justifier le passage de la relativité restreinte a la générale, s'est appuyé, en premiere ligne, sur un motif gnoséologique, auquel il a donné une signification décisive, a côté des fondements purement physico-empiriques. » (CASSIRER, 1994, p. 7).

Une lecture «néo-kantienne » de la relativité de Einstein découvre maintenant son programme. Il ne s'agit pas de comparer les theses de la nouvelle physique avec les theses kantiennes positives de la Critique ou meme des Premiers principes métaphysiques de la science de la nature, comme si une intégration ou une adaptation était possible ou désirable. Il s'agit, plutôt, de s'engager dans cette zone de confluence entre science de la Nature et gnoséologie afin de déterminer en quelle mesure la compréhension kantienne de la dynamique d'objectivation, constitutive de l'objectivité d'expérience, est encore suffisamment puissante pour bien comprendre le processus de formation interne des concepts théoriques de la nouvelle physique et des theses positives qui en dérivent. Telle est la question en laquelle se décide l'actualité ou l'obsolescence de la philosophie critique de Kant.

\section{L'esthétique transcendantale dans un carrefour}

Toute la stratégie argumentative de Cassirer se concentre sur les deux points suivants : en premier lieu, présentation du principe de la covariance, de la relativité généralisée, non comme une simple these empirique concernant le statut des objets physiques, mais comme un principe synthétique, constitutif, que l'entendement prescrit pour l'interprétation des phénomenes de la Nature ; en deuxieme lieu, affaiblissement des theses de l'esthétique, en distinguant entre théorie transcendantale de l'espace et du temps en tant que méthodes d'objectivation et théorie empirique de l'espace et du temps en tant que grandeurs physiques, cherchant a situer au niveau du probleme "empirique » de la métrique les changements que la théorie de la relativité a introduit.

Contre cette interprétation, nous voudrions montrer que le principe de la covariance, que Cassirer reconnaît justement comme le noyau de la théorie relativiste de l'objectivité physique, agit en retour sur la conception kantienne de l'espace et du temps de l'esthétique et demande, par lui seul, une " esthétique transcendantale » de type nouveau, organisée autour de problemes et d'objectifs différents.

Le principe de la covariance générale - ce que Einstein désigne comme « postulat de la relativité générale »- 
prescrit que " les lois physiques doivent etre d'un type tel qu'elles se peuvent appliquer a des systemes de référence en toutes sortes de mouvement. »ou, dans une autre formulation, que " les lois de la Nature doivent etre exprimées par des équations qui soient valides pour tous les systemes de coordonnées, c'est a dire, qui soient covariantes relativement a tous les remplacements arbitraires (généralement covariantes). »(EINSTEIN, 1916, p. 769 822) En un mot, le postulat de la relativité générale, lui-meme une généralisation du principe de la relativité restreinte pour systemes inertiels, déclare que toutes les coordonnées gaussiennes (donc, aussi celles des systemes accélérés, identifiés la chute libre des corps dans un champ gravitationnel par le " principe d'équivalence ») de l'espacetemps sont équivalentes pour la formulation des lois de la Nature.

Comme on l'admet communément, la these de la covariance suppose que :

1. Les mesures spatiales et temporelles (soit les distances d'espace et de temps entre deux points donnés) des événements ne sont pas invariantes - au contraire, elles dépendent du systeme de référence arbitrairement choisi et, donc, de la situation particuliere de l'observateur (idéalisé en la forme d'un systeme regle-chronometre);

2. Mais les lois de la Nature, en étant énoncées par des équations différentielles généralement covariantes, expriment en leur forme l'indépendance du point de vue particulier de l'observateur, et c'est justement cette indépendance qui les qualifie en tant que lois de la Nature;

3. Aucun objet empirique ne peut etre un référentiel absolument privilégié (soit la Terre immobile, le centre de rotation du Soleil ou l'éther), mais tous les référentiels pour les mesures d'espace et de temps se transforment les uns dans les autres et l'unique référence absolue est la loi de transformation elle-meme.

Le commentaire de Cassirer est incisif. En paraphrasant Max Planck, il dira que, avec le postulat de la covariance, " l'anthropomorphisme de l'image sensible naturelle du monde, dont le dépassement est la tâche propre de la connaissance physique, a été, ici, poussée en arriere un pas de plus. " (CASSIRER, 1994, p. 37) Or cela se vérifie justement parce que le postulat de la covariance vient exprimer un concept plus profond d'objectivité, en la pensant non par rapport aux particularités de l'intuition sensible d'un sujet épistémique déterminé, mais par rapport á l'idée de la totalité des points de vue possibles et a ce que, dans la variation arbitraire de ces points de vue, reste cependant invariable. Cet invariant n'est pas un autre point de vue, "absolu", au-dela de la multiplicité des observa- 
teurs, une espece de systeme de référence privilégié, mais constitue, en revanche, la position d'un réel, en soimeme pleinement déterminé, dont le corrélat épistémique est l'intégral de ces variations possibles (connectés selon une loi de transformation) par rapport a une multiplicité ouvert de tous les référentiels possibles. Il ne s'agit, ici, d'une simple sophistication mathématique des équations qui expriment les lois de la Nature (a laquelle, par exemple, les équations de Newton ne résistent pas). Il s'agit plutôt de la mise en scene d'un concept plus puissant en ce qui concerne ce qu'on doit entendre par objet de la science de la Nature. Ce nouveau concept, s'il nous libere, comme Cassirer le dit, de l'image anthropoforme du monde, construite dans l'élément de la représentation sensible immédiate, est, de l'autre côté, un développement nouveau du principe kantien de l'objectivité en tant que corrélat de "l'unité synthétique » d'un divers d'intuitions possibles. Dans l'interprétation de Cassirer, la covariance répond a l'idée kantienne de l'unité de la Nature, de la détermination univoque - « objet » est, ici, le résultat d'une synthese que se réalise par rapport a la diversité des observateurs possibles et qui pose comme unité un réel en tant qu'invariant de cette variation. Ce concept nouveau au sujet de l'objectivité physique ne se rapporte pas a quelque chose d'immédiatement donné dans une expérience empiri- que particuliere, mais il est capable d'exprimer quelque chose comme une loi de la Nature uniquement en faisant varier arbitrairement les coordonnées spatiales et temporales de l'expérience immédiate et en fixant un invariant en tant que regle de transformation de toutes les coordonnées possibles. En ce sens, comme Cassirer le remarque, qu'une "loi " soit un tel invariant pour toutes les transformations arbitraires de coordonnées, cela est certainement une proposition analytique, mais qu'il y a en général de telles lois, que l'entendement doive chercher la forme de l'invariance pour exprimer une connaissance d'objet, cela est une exigence synthétique, c'est a dire, un principe constitutif que l'entendement prescrit pour l'interprétation des phénomenes naturels - "Nous pouvons seulement désigner comme lois de la Nature, c'est a dire, leur attribuer universalité objective, ces relations dont la forme est indépendante de notre mesure empirique, du choix spécifi$\begin{array}{lllll}\text { que des quatre variables } & \mathrm{x}_{1} & \mathrm{x}_{2} & \mathrm{x}_{3} & \mathrm{x}_{4}\end{array}$ qu'expriment les parametres d'espace et de temps. En ce sens, le principe de la relativité générale, selon lequel les lois de la Nature ne changent pas par une transformation completement arbitraire des variables spatio-temporelles, peut etre entendu comme une affirmation analytique, comme un éclaircissement de ce que nous devons entendre par une loi "générale " de la Nature - synthétique est, pourtant, l'exigence 
qu'il doit y avoir, en général, de tels invariants ultimes. " (CASSIRER, 1994, p. 37)

Disons, cependant, que ce postulat de l'invariance peut seulement etre interprété de façon kantienne au prix d'une profonde réinterprétation de Kant lui-meme. L'unité de la Nature, ou le principe de la "détermination univoque ", surgit en tant que corrélat non pas d'une subjectivité transcendantale qui doit opérer la synthese par rapport a ce que lui est donné dans l'intuition empirique, comme si les mesures d'espace et de temps étaient des données absolues, invariables pour tout sujet épistémique, a subsumer simplement sous les conditions catégorielles, logiques, de la représentation objective, mais le sujet transcendantal est le lieu de droit pour la formulation de lois qui, en étant généralement covariantes, doivent non seulement faire abstraction de la particularité de l'intuition sensible, mais aussi soumettre dorénavant la forme esthétique elle-meme (c'est a dire, la théorie des grandeurs d'espace et de temps) aux exigences logiques de la représentation objective. On ne va pas de l'esthétique a l'analytique; au contraire, la théorie des formes esthétiques doit etre déja une fonction de la théorie catégorielle de la pensée objective.

Cette subordination de l'esthétique a la logique transcendantale est, d'ailleurs, une des vertus majeures de l'école de Marburg et un héritage ferme que Cassirer prend de Cohen et de Natorp. Essayons quelques pas en avant, autrement et au-dela de la stratégie purement défensive de Cassirer, bâtie sur la distinction entre enquete transcendantale sur l'espace et le temps pur (celle de Kant) et théorie empirique de la mesure de l'espace et du temps physiques (celle de Einstein), stratégie qui se heurte a l'objection évidente que la théorie kantienne de l'espace et du temps, qui est aussi une théorie de l'espace et du temps en tant que déterminations de l'objet empirique, est inapplicable a la nouvelle physique, organisé autour d'une théorie de l'objectivité physique qui fait appel a une doctrine de l'espace-temps (non de l'espace et du temps) et a la métrique tout a fait nouvelle qui en résulte.

\section{Quelques problemes pour une esthétique transcendantale nouvelle}

Quelles que soient les lois empiriques que la connaissance de la Nature peut établir, le postulat de la covariance, interprété comme un principe transcendantal constitutif regardant la forme de toute loi possible (la natura formaliter spectata), demande a l'esthétique une théorie de l'espace, du temps et de la détermination spatio-temporelle des phénomenes qui soit compatible avec le principe suivant : bâtir une théorie de l'espace-temps, en tant que grandeur mathématico-physique, tel que les phé- 
nomenes empiriques puissent etre univoquement localisables pour tout changement arbitraire des coordonnées qui s'enracinent en la position particuliere d'un l'observateur. C'est cette localisation univoque des phénomenes qui permettra la forme covariante des lois de 1 '« entendement. » Le probleme de l'esthétique serait, ainsi, le probleme (déja kantien) de l'individuation par rapport a l'espace-temps ou de l'espacetemps en tant que conditions de possibilité de l'individuation en général.

Cela exigera :

1. Un changement en profondeur des concepts fondamentaux de l'esthétique. On doit, en premier lieu, substituer la théorie kantienne et newtonienne de l'espace et du temps, laquelle est, en vérité, une théorie de l'espace a travers le temps, par une théorie de l'espacetemps en tant que continuum quadridimensionnel. Kant lui-meme a donné quelques pas hésitants dans la direction d'une unité de ces deux représentations. D'un côté, il a soutenu que la représentation de l'espace, en tant que condition du sens externe, est dépendante de la représentation du temps, en tant que forme du sens interne. De l'autre côté, surtout a propos de la " réfutation de l'idéalisme ", il a aussi soutenu, cependant, que la détermination de temps (c'est a dire, la conscience empiriquement déterminée de ma propre existence) est seulement possible par rapport a un permanent donné dans l'espace. Du croisement de ces deux arguments, qui vont en directions opposées, on sera tenté de conclure qu'espace et temps sont des représentations co-originaires, qui s'impliquent mutuellement. Kant ne suggere pas, cependant, que les représentations de l'espace et du temps s'entremelent dans la représentation unitaire et seulement concrete de l'espace-temps. La théorie des formes du sens interne et externe offre une origine différente pour chacune de ces représentations. Elles sont séparées - il y a le temps et l'espace, et chacune de ces représentations a une unité propre en tant que représentation singuliere. Malgré cela, si on regarde du côté des objets de la Nature, donnés dans une intuition empirique, on voit les déterminations d'espace et de temps se superposer : tout objet dans l'espace est donné a un moment du temps et tout moment du temps est donné par l'entremise d'un objet dans l'espace, d'une façon telle que l'expérience des objets physiques ne nous donne aucunement l'espace et en plus le temps, mais l'union des deux, c'est a dire, quelque chose comme la grandeur une et unique de l'espacetemps. En deuxieme lieu, par rapport aux points de l'espace-temps, qui se définissent par des coordonnées spatiales et temporelles, on aura dans le concept d'événement le premier concept concret de l'esthétique. "Evénement » désigne non pas seulement ce que remplit un espace, mais quelque chose qui a lieu ou qui arrive dans l'espace-temps. Afin 
de donner les coordonnées d'un événement, on doit parler unitairement de sa localisation spatio-temporelle. A propos de ce que remplit l'espace et le temps dans une intuition empirique, Kant parle simplement de Erscheinung, c'est a dire, d'apparition. On doit penser l'apparition comme événement et redéfinir l'esthétique. La théorie des conditions de possibilité de l'intuition d'objets en général deviendra une théorie des conditions de possibilité de la localisation spatio-temporelle univoque des événements pour tout observateur possible.

2. Une réélaboration de la métrique de l'espace-temps. On a le concept géométrique de "distance » entre deux points de l'espace. En ce qui concerne la grandeur mathématico-physique (pas simplement "géométrique ») de l'espacetemps, le concept de distance entre deux points » implique non seulement une valeur différente de zéro pour les variables spatiales, mais aussi une valeur différente de zéro pour la variable temporelle. Si on pense géométriquement la distance entre deux points, on considere tous les points de l'espace comme donnés dans le meme instant. En revanche, le concept mathématicophysique d'espace-temps implique que la distance entre deux points soit a la fois spatiale et temporelle. En pensant a un parcours unissant deux points de l'espace-temps, nous devrons spécifier, donc, combien d'espace en combien de temps nous permettra d'arriver au deu- xieme point en partant du premier. On doit, ainsi, parler d'une vitesse $(\mathrm{d} / \mathrm{t})$ pour définir la translation d'un point $A$ sur un point $B$ et, en particulier, on peut faire la supposition d'une vitesse maximale, $V$. Si, au contraire, on admet que $V$ peut s'accroître a l'infini, on arrive au concept d'une connexion entre deux événements a distance dans un temps égal a zéro, c'est a dire, on obtient les concepts de simultanéité absolue entre deux événements a distance et d'une connexion qui se fait dans l'instant. En un mot: on arrive aux conceptions de Newton et de Kant. Mais, comme Minkowski l'a bien souligné, la supposition selon laquelle $V=\infty$ est " moins intelligible » que l'hypothese selon laquelle $V=c$, « $c$ » étant une quantité finie qu'on doit définir empiriquement (soit la vitesse de la lumiere dans de vide). Par ailleurs, les équations de la mécanique de Newton ne sont pas invariantes pour toutes les transformations arbitraires de coordonnées spatiotemporelles. Si, en conformité avec les exigences « logiques »de l'invariance pour les lois de la Nature, on admet la supposition "plus intelligible » selon laquelle la «distance » (on parlera d' « intervalle ») entre points de l'espacetemps implique que $V$ soit finie et la meme pour tous les observateurs (en dépit de son état de mouvement relatif), on obtiendra par-la la relativité de la simultanéité et les effets relativistes de la "dilatation » du temps et de la « contraction» de l'espace pour obser- 


\section{vateurs en mouvement les uns par rap- trouvera la structure mathématique de port aux autres. En un mot, on re- l'espace-temps de Minkowski.}

\section{Bibliographie}

CASSIRER, Ernst. Substanzbegriff und Funktionsbegriff. Untersuchungen über die Grundfragen der Erkenntnistheorie. Berlin, 1910. Substance et fonction. Éléments pour une théorie du concept. Paris : Les Éditions de Minuit, 1977.

. Zur Einsteinschen Relativitätstheorie. Erkenntnistheoretische Betrachtungen. Berlin: Bruno Cassirer Verlag, 1921. République en Zur modernen Physik. Darmstadt: W.B., 1994, pp. 1-125.

EINSTEIN, Albert. Die Grundlage der allgemein Relativitätstheorie" , Annalen der Physik 49, 1916, pp. 769-822.

FRIEDMAN, Michael. Dynamics of Reason. Standford: CSLI Publications, 2001.

REICHENBACH, Hans. Relativitätstheorie und Erkenntnis A Priori . Berlin: J. Springer, 1920.

. The Philosophical Significance of the Theory of Relativity. In Albert Einstein. Philosopher-Scientist. Third edition. La Salle, Illinois: Open Court Publishing Company, 2000.

RYCKMAN, Thomas. The Reign of Relativity. Philosophy in Physics 1915-1925. Oxford: Oxford University Press, 2005.

Received / Recebido: 18/07/2020

Approved / Aprovado: 18/11/2020

Published / Publicado: 30/12/2020 
